\title{
The Efficient Proportional Myerson Values for Hypergraph Games
}

\author{
Guangming Wang $\mathbb{D}^{1,2}$ Lei Cai, ${ }^{1}$ and Erfang Shan $\mathbb{D}^{1}$ \\ ${ }^{1}$ School of Management, Shanghai University, Shanghai 200444, China \\ ${ }^{2}$ School of Mathematical Sciences, University of Jinan, Jinan 250022, China \\ Correspondence should be addressed to Erfang Shan; efshan@shu.edu.cn
}

Received 6 May 2021; Accepted 15 June 2021; Published 29 June 2021

Academic Editor: Cuimei Jiang

Copyright ( 2021 Guangming Wang et al. This is an open access article distributed under the Creative Commons Attribution License, which permits unrestricted use, distribution, and reproduction in any medium, provided the original work is properly cited.

\begin{abstract}
This study deals with a class of efficient extensions of Myerson value for games with hypergraph communication situations in which the surplus is allocated proportionally. We introduce $w$-fairness of surplus and provide axiomatic characterizations of the new allocation rule. Furthermore, we give an example of research fund distribution amongst researchers, compare the numerical results with several values, and realize other efficient extensions of Myerson value can be obtained depending on the different measure function $w$ on the hypergraph.
\end{abstract}

\section{Introduction}

Cooperative games with transferable utility (TU-game) [1] usually describe situations in which a viable alliance can be formed and earn the corresponding worth by cooperating. However, in many situations, viable alliance is restricted by some hierarchical, cultural, or communicational technology constraints. For this reason, Myerson [2] introduced the Myerson value which is a kind of Shapley value [3] depended on graph and characterized by component efficiency and fairness. Later, Myerson [4] and Slikker and van den Nouweland [5] studied other axiomatic characterizations of the Myerson value. In recent years, several works have devoted to the study of Myerson value. Li and Shan [6-8] studied the Myerson value on structures of coalitions or for directed graph games. van den Nouweland [9] and Shan and Zhang [10] extended the idea initiated by Myerson to hypergraph games.

The Myerson value satisfying component efficiency, i.e., the worth of every component, is distributed among its members. However, in many situations, every player obtains distribution of the worth generated by the grand coalition such as research fund distribution amongst researchers. Motivated by this idea, van den Brink [11] introduced the efficient egalitarian Myerson value (EEMy) for graph games which is the first efficient extension of the Myerson value. Beal [12] proved that EEMy is the unique efficient and fair extension of Myerson value. Casajus [13-16] introduced the efficient two-step egalitarian surplus Myerson value (ESMy) on graph communication situations which is the first nonfair efficient extension of the Myerson value. However, an axiomatic characterization of the efficient extension of the Myerson value for hypergraph games remains an open problem. Motivated by the above discussion, we investigate a class of efficient extensions of the Myerson value for games with hypergraph communication situations in which the surplus is allocated proportionally.

The rest of this paper is organized as follows. In Section 2, we provide some preliminaries on TU-games, the graph, and the hypergraph games' allocation rule. In Section 3, we propose a class of efficient extensions of the Myerson value, provide a characterization of $E^{w} M y$, and give an example of research fund distribution amongst researchers. Section 4 concludes with some remarks.

\section{Preliminaries}

A transferable utility game (TU-game) represented by a pair $(N, v)$, where $N=\{1,2, \ldots, n\}$, is a set of players and $v: 2^{N} \longrightarrow \mathbb{R}$ is a characteristic function with $v(\varnothing)=0$. An 
allocation rule (A value) is a function about the payoff of player in a TU-game. A very famous value is the Shapley value: $\quad \operatorname{Sh}_{i}(v)=\sum_{S \subseteq N} \quad(|N|-|S|) !(|S|-1) ! /|N| !(v(S)-v$ $(S \backslash\{i\})), i \in N$.

The triple $(N, v, H)$ represents a hypergraph games, which is composed of two parts: the TU-game $(N, v)$ and the hypergraph structure $(N, H)$ on $N$, where $H \subseteq H^{N}:=$ $\{e \subseteq N|| e \mid \geq 2\}$; i.e., $H$ is a family of nonsingleton subsets of $N$, called hyperlinks. In particular, a hypergraph $(N, H)$ is a graph if $|e|=2$.

For each player $i \in N, H_{i}:=\{e \in H \mid i \in e\}$ is the set of hyperlinks containing $i$ in $(N, H)$. A node $i \in N$ is incident to a hyperlink $e \in H$ if $i \in e$. Two nodes $i, j \in N$ are adjacent if there is a hyperlink $e$ in $H$ satisfying $i, j \in e$. We say that nodes $i$ and $j$ are connected in $(N, H)$ if there exists is a sequence $i=i_{1}, e_{1}, i_{2}, e_{2}, \ldots, i_{k}, e_{k}, i_{k+1}=j$ such that $i_{l}, i_{l+1} \in e_{l}$, for $l=1,2, \ldots, k$. A hypergraph is connected if every pair of nodes is connected. Connectedness in $(N, H)$ induces a partition of $N$ into components. A component is a maximal set of nodes of $N$ in which every pair of nodes are connected. Let $N / H$ be the set of components of $(N, H)$ and $(N / H)_{i}$ be the component containing $i \in N$. We denote by $\mathrm{HG}$ the set of all hypergraph games and $G$ the set of all graph games. Hypergraph (graph) games are called connected if the associated hypergraph (graph) is connected. We denote, by $\mathrm{HG}_{C}$, the class of all connected hypergraph games and, $G_{C}$, the class of all connected graph games.

An allocation rule or value $f(N, v, H)$ on hypergraph communication situations is a $n$-dimensional vector function defined on hypergraph games. The Myerson value for hypergraph communication situations is defined as follows:

$$
\operatorname{My}_{i}(N, v, H)=\operatorname{Sh}_{i}\left(N, v^{H}\right), \quad \text { for any } i \in N
$$

where $v^{H}(S)=\sum_{T \in S / H} v(T)$ for any $S \subseteq N$. The game $\left(N, v^{H}\right)$ is called the point game.

The Myerson value is the unique hypergraph games' allocation rule which satisfies component efficiency (CE) and fairness $(\mathbf{F})$.

Component efficiency (CE): For any $(N, v, H) \in \mathrm{HG}$ and $C \in N / H$,

$$
\sum_{i \in C} f_{i}(N, v, H)=v(C) .
$$

Fairness (F): For any $(N, v, H) \in \mathrm{HG}$ and $i j \in H$,

$$
\begin{aligned}
f_{i}(N, v, H)-f_{i}(N, v, H \backslash\{i j\})= & f_{j}(N, v, H) \\
& \left.-f_{j}(N, v, H \backslash i j\}\right) .
\end{aligned}
$$

More axioms for efficiency extensions of the Myerson value are as follows.
Efficiency $(\mathbf{E})$ : For any $(N, v, H) \in \mathrm{HG}$,

$$
\sum_{i \in N} f_{i}(N, v, H)=v(N) .
$$

Coherence with the Myerson value for connected hypergraphs (CMC): For any $(N, v, H) \in \mathrm{HG}_{C}$,

$$
f(N, v, H)=\operatorname{My}(N, v, H) \text {. }
$$

\section{A Class of Efficient Extensions of Myerson Value for Hypergraph Games}

In this section, we propose a class of efficient extensions of the Myerson value which distributes the surplus of the Myerson value by defining some measure function $w$ on hypergraph. Let $(N, v, H)$ be any hypergraph games and let us define a function $w:(N, H) \longrightarrow R$ which assigns to every player $i \in N$, a real number $w_{i}(N, H)$. We define the efficient $w$ - proportional Myerson value $E^{w} \mathrm{My}$ :

$$
\begin{aligned}
E^{w} \operatorname{My}_{i}(N, v, H)= & \operatorname{My}_{i}(N, v, H) \\
& +\frac{w_{i}(N, H)}{\sum_{k \in N} w_{k}(N, H)}\left[v(N)-v^{H}(N)\right] .
\end{aligned}
$$

In order to characterize efficient $w$-proportional Myerson value $M$ yerson value $E^{w} \mathrm{My}$, we propose the following property.

$w$-fairness of surplus $\left(\mathbf{F S}^{w}\right)$ : a value on HG satisfies $\mathbf{F S}^{w}$ if, for any $(N, v, H) \in \mathrm{HG}$ and any $i, j \in N$,

$$
\begin{aligned}
& \frac{1}{w_{i}(N, H)}\left[\xi_{i}(N, v, H)-\xi_{i}\left(C_{i}, v_{C_{i}}, H_{C_{i}}\right)\right] \\
& =\frac{1}{w_{j}(N, H)}\left[\xi_{j}(N, v, H)-\xi_{j}\left(C_{j}, v_{C_{j}}, H_{C_{j}}\right)\right] .
\end{aligned}
$$

Then, we provide a characterization of $E^{w} \mathrm{My}$.

Theorem 1. The efficient extension Myerson value $E^{w} M y$ on hypergraph communication situations is the unique allocation which satisfies efficiency $(E), w$-fairness of surplus $\left(\mathbf{F S}^{w}\right)$, and coherence with the Myerson value for connected hypergraphs (CMC).

Proof. (existence). Let $(N, v, H)$ is any hypergraph games. It is easy to see that $E^{w} \mathrm{My}(N, v, H)$ satisfies $\mathbf{E}$ and $\mathbf{C M C}$.

Next, we prove that $E^{w} \operatorname{My}(N, v, H)$ satisfies w-fairness of surplus $\left(\mathbf{F S}^{w}\right)$. By the definition of $E^{w} \mathrm{My}(N, v, H)$, we have 


$$
\begin{aligned}
& \frac{1}{w_{i}(N, H)}\left[E^{w} \operatorname{My}_{i}(N, v, H)-E^{w} \operatorname{My}_{i}\left(C_{i}, v_{C_{i}}, H_{C_{i}}\right)\right] \\
& =\frac{1}{w_{i}(N, H)}\left[\operatorname{My}_{i}(N, v, H)+\frac{w_{i}(N, H)}{\sum_{k \in N} w_{k}(N, H)}\left(v(N)-v^{H}(N)\right)-\mathrm{My}_{i}\left(C_{i}, v_{C_{i}}, H_{C_{i}}\right)\right] \\
& =\frac{1}{w_{i}(N, H)}\left[M y_{i}\left(C_{i}, v_{C_{i}}, H_{C_{i}}\right)+\frac{w_{i}(N, H)}{\sum_{k \in N} w_{k}(N, H)}\left(v(N)-v^{H}(N)\right)-\mathrm{My}_{i}\left(C_{i}, v_{C_{i}}, H_{C_{i}}\right)\right] \\
& =\frac{1}{\sum_{k \in N} w_{k}(N, H)}\left(v(N)-v^{H}(N)\right),
\end{aligned}
$$

where the second equality follows from the component decomposability of the Myerson value. Similarly, we have

$$
\begin{aligned}
& \frac{1}{w_{j}(N, H)}\left[E^{w} \operatorname{My}_{j}(N, v, H)-E^{w} \operatorname{My}_{j}\left(C_{j}, v_{C_{j}}, H_{C_{j}}\right)\right] \\
& =\frac{1}{w_{j}(N, H)}\left[\operatorname{My}_{j}(N, v, H)+\frac{w_{j}(N, H)}{\sum_{k \in N} w_{k}(N, H)}\left(v(N)-v^{H}(N)\right)-\mathrm{My}_{j}\left(C_{j}, v_{C_{j}}, H_{C_{j}}\right)\right] \\
& =\frac{1}{w_{i}(N, H)}\left[M y_{j}\left(C_{j}, v_{C_{j}}, H_{C_{j}}\right)+\frac{w_{j}(N, H)}{\sum_{k \in N} w_{k}(N, H)}\left(v(N)-v^{H}(N)\right)-\operatorname{My}_{j}\left(C_{j}, v_{C_{j}}, H_{C_{j}}\right)\right] \\
& =\frac{1}{\sum_{k \in N} w_{k}(N, H)}\left(v(N)-v^{H}(N)\right) .
\end{aligned}
$$

Therefore, we obtain

$$
\begin{aligned}
& \frac{1}{w_{i}(N, H)}\left[E^{w} \operatorname{My}_{i}(N, v, H)-E^{w} \mathrm{My}_{i}\left(C_{i}, v_{C_{i}}, H_{C_{i}}\right)\right] \\
& =\frac{1}{w_{j}(N, H)}\left[E^{w} \operatorname{My}_{j}(N, v, H)-E^{w} \operatorname{My}_{j}\left(C_{j}, v_{C_{j}}, H_{C_{j}}\right)\right] .
\end{aligned}
$$

Hence, $E^{w} \mathrm{My}_{i}(N, v, H)$ satisfies $\mathbf{F S}^{w}$. To show uniqueness and recall $\mathbf{F} \mathbf{S}^{w}$,

$$
\begin{aligned}
& \frac{1}{w_{i}(N, H)}\left[\xi_{i}(N, v, H)-\xi_{i}\left(C_{i}, v_{C_{i}}, H_{C_{i}}\right)\right] \\
& =\frac{1}{w_{j}(N, H)}\left[\xi_{j}(N, v, H)-\xi_{j}\left(C_{j}, v_{C_{j}}, H_{C_{j}}\right)\right]=t .
\end{aligned}
$$

There exists a constant $t$, such that $\xi_{i}(N, v, H)-\xi_{i}\left(C_{i}, v_{C_{i}}, H_{C_{i}}\right)=t w_{i}(N, H), \quad$ for all $i \in N$.

We have

$$
\xi_{i}(N, v, H)=\xi_{i}\left(C_{i}, v_{C_{i}}, H_{C_{i}}\right)+t w_{i}(N, H)
$$

By summing up over all $i \in N$, we obtain

$$
\sum_{i \in N} \xi_{i}(N, v, H)=\sum_{i \in N} \xi_{i}\left(C_{i}, v_{C_{i}}, H_{C_{i}}\right)+t \sum_{i \in N} w_{i}(N, H)
$$

By efficiency, $\quad \sum_{i \in N} \xi_{i}(N, v, H)=v(N) \quad$ and $\sum_{i \in N} \xi_{i}\left(C_{i}, v_{C_{i}}, H_{C_{i}}\right)=v^{H}(N)$. Thus,

$$
v(N)=v^{H}(N)+t \sum_{i \in N} w_{i}(N, H) .
$$

Then, $t=1 / \sum_{i \in N} w_{i}(N, H)\left[v(N)-v^{H}(N)\right]$. By equation (13), we obtain

$$
\begin{aligned}
\xi_{i}(N, v, H)= & \xi_{i}\left(C_{i}, v_{C_{i}}, H_{C_{i}}\right) \\
& +\frac{w_{i}(N, H)}{\sum_{i \in N} w_{i}(N, H)}\left[v(N)-v^{H}(N)\right] .
\end{aligned}
$$

Since $\xi$ satisfies CMC, then $\xi_{i}\left(C_{i}, v_{C_{i}}, H_{C_{i}}\right)=\operatorname{My}_{i}\left(C_{i}\right.$, $\left.v_{C_{i}}, H_{C_{i}}\right)$. Furthermore, $\operatorname{My}_{i}\left(C_{i}, v_{C_{i}}, H_{C_{i}}\right)=\operatorname{My}_{i}(N, v, H)$ because of component decomposability of the Myerson value. Hence,

$$
\begin{aligned}
\xi_{i}(N, v, H)= & \mathrm{My}_{i}(N, v, H) \\
& +\frac{w_{i}(N, H)}{\sum_{i \in N} w_{i}(N, H)}\left[v(N)-v^{H}(N)\right] .
\end{aligned}
$$

Obviously, $\xi_{i}(N, v, H)=E^{w} \mathrm{My}_{i}(N, v, H)$, as desired. 
In particular, when $w_{i}(N, H)=c(c$ is nonzero constant $)$, $w$-fairness of surplus is reduced to fairness of surplus:

$$
\begin{aligned}
f_{i}(N, v, H)-f_{i}\left(C_{i}, v_{C_{i}}, H_{C_{i}}\right)= & f_{j}(N, v, H) \\
& -f_{j}\left(C_{j}, v_{C_{j}}, H_{C_{j}}\right) .
\end{aligned}
$$

and

$$
\begin{aligned}
& E^{w} \operatorname{My}_{i}(N, v, H): \\
& =\operatorname{My}_{i}(N, v, H)+\frac{w_{i}(N, H)}{\sum_{k \in N} w_{k}(N, H)}\left[v(N)-v^{H}(N)\right] \\
& =\operatorname{My}_{i}(N, v, H)+\frac{c}{|N| c}\left[v(N)-v^{H}(N)\right] \\
& =\operatorname{My}_{i}(N, v, H)+\frac{v(N)-v^{H}(N)}{|N|} \\
& =\operatorname{EEMy}_{i}(N, v, H) .
\end{aligned}
$$

Theorem 2. The efficient egalitarian Myerson value EEMy on hypergraph communication situations is the unique allocation satisfy efficiency (E), fairness of surplus (FS), and coherence with the Myerson value for connected hypergraphs (CMC).

Furthermore, when restrict hypergraph communication situations $(N, v, H)$ to graph communication situations $(N, v, L)$ and $w_{i}(N, H)=c$, we obtain the conclusion of van den Brink et al. [11] as an immediate consequence of Theorem 1:

$$
\operatorname{EEMy}_{i}(N, v, L):=\operatorname{My}_{i}(N, v, L)+\frac{v(N)-v^{L}(N)}{|N|},
$$

Theorem 3. The efficient egalitarian Myerson value EEMy on graph communication situations is the unique allocation satisfy efficiency (E), fairness of surplus (FS), and coherence with the Myerson value for connected graphs (CMC).

When $w_{i}(N, H)=1 /\left|C_{i}\right|, w$ - fairness of surplus is reduced to per capita fairness of surplus:

$$
\begin{aligned}
& \left|C_{i}\right|\left(f_{i}(N, v, H)-f_{i}\left(C_{i}, v_{C_{i}}, H_{C_{i}}\right)\right) \\
& \quad=\left|C_{j}\right|\left(f_{j}(N, v, H)-f_{j}\left(C_{j}, v_{C_{j}}, H_{C_{j}}\right)\right)
\end{aligned}
$$

and

$$
\begin{aligned}
& E^{w} \operatorname{My}_{i}(N, v, H): \\
& =\operatorname{My}_{i}(N, v, H)+\frac{1 /\left|C_{i}\right|}{\sum_{k \in N} 1 /\left|C_{k}\right|}\left[v(N)-v^{H}(N)\right] \\
& =\operatorname{My}_{i}(N, v, H)+\frac{v(N)-v^{H}(N)}{|N / H|\left|C_{i}\right|} \\
& =\operatorname{ESMy}_{i}(N, v, H)
\end{aligned}
$$

Theorem 4. The efficient two-step egalitarian surplus Myerson value ESMy on hypergraph communication situations is the unique allocation satisfy percapita fairness of surplus fairness of surplus (PRFS), efficiency (E), and coherence with the Myerson value for connected hypergraphs (CMC).

Furthermore, when restricting hypergraph communication situations $(N, v, H)$ to graph communication situations $(N, v, L)$ and $w_{i}(N, H)=1 /\left|C_{i}\right|$, we obtain the conclusion of Casajus which introduced the efficient twostep egalitarian surplus Myerson value(ESMy) on graph communication situations [13] as another immediate consequence of Theorem 1:

$$
\operatorname{ESMy}_{i}(N, v, L):=\operatorname{My}_{i}(N, v, L)+\frac{v(N)-v^{L}(N)}{|N / L||T|} .
$$

Theorem 5. The efficient two-step egalitarian surplus Myerson value ESMy on graph communication situations is the unique allocation which satisfies percapitafairness of surplus of surplus (PRFS), efficiency (E), and coherence with the Myerson value for connected graphs (CMC).

Example 1. Consider a problem of research fund distribute amongst researchers. Suppose fund total amounts is $4, A$ is a researcher of subject one, $D$ is a researcher of subject two, $B, C$ are the researchers of subject one and subject two, $E$ is a researcher of subject there, and hypergraph communication situations is $(N, v, H)$ with Figure 1 , where $N=\{A, B$, $C, D, E\}, \quad H=\{\{A, B, C\},\{B, C, D\}, \quad$ and $\quad v(S)=|S|-1$, $|S|=1,2,3,4,5$.

The Shapley value of $v$ is efficient and $\operatorname{Sh}(v)=(4 / 5,4 / 5,4 / 5,4 / 5,4 / 5)$. The Myerson value is component efficient and $\operatorname{My}(v, H)=(2 / 3,5 / 6,5 / 6,2 / 3,0)$. Although fund amounts is 4 , by component efficient, the total amount of the Myerson value is 3. Fund surplus is $v(N)-v^{H}(N)=4-3=1$.

On the one hand, $v(N)-v^{H}(N) /|N|=1 / 5$, efficient $w-$ proportional Myerson value $E^{w} \mathrm{My}\left(w_{i}(N, H)=c\right)$ reduces to efficient egalitarian Myerson value EEMy, i.e., $E^{w} \mathrm{My}$ $(v, H)=\operatorname{EEMy}(v, H)=(13 / 15,31 / 30,31 / 30,13 / 15,1 / 5)$, the allocation satisfy FS: $f_{i}(N, v, H)-f_{i}\left(c_{i}, v_{c_{i}}, H_{c_{i}}\right)=$ $f_{j}(N, v, H)-f_{j}\left(c_{j}, v_{c_{j}}, H_{c_{j}}\right)=1 / 5$. 


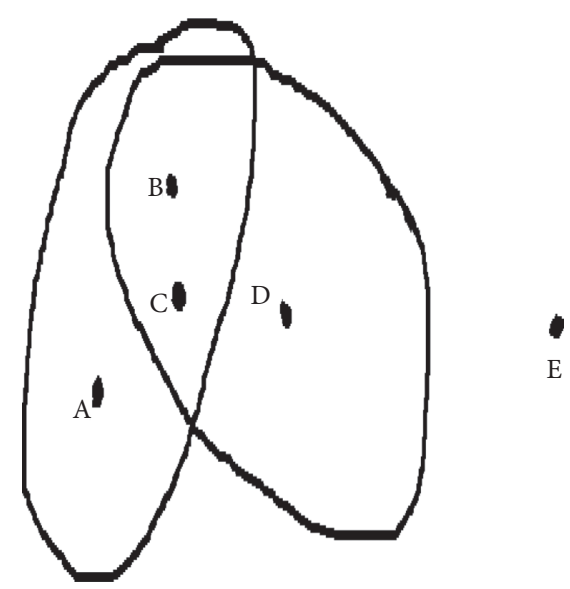

Figure 1: Fund distribution amongst researchers.

On the other hand, $v(N)-v^{H}(N) /|N / H|\left|C_{i}\right|=$ $1 / 2 * 4, i=A, B, C, D, v(N)-v^{H}(N) /|N / H|\left|C_{i}\right|=1 / 2 * 1$, $i=E$, and efficient $w$ - proportional Myerson value $E^{w} \operatorname{My}\left(w_{i}(N, H)=1 /\left|C_{i}\right|\right)$ reduce to efficient two-step egalitarian surplus Myerson value ESMy, i.e., $E^{w} \mathrm{My}(v, H)=$ $\operatorname{ESMy}(v, H)=(19 / 24,23 / 24,23 / 24,19 / 24,1 / 2)$, and the allocation satisfies PRFS: $\left|C_{i}\right|\left(f_{i}(N, v, H)-f_{i}\left(c_{i}, v_{c}, H_{c_{i}}\right)\right)=$ $\left|C_{j}\right|\left(f_{j}(N, v, H)-f_{j}\left(c_{j}, v_{c_{j}}, H_{c_{j}}\right)\right), 4 * 1 / 8=1 * 1 / 2=1 / 2$.

\section{Conclusion}

In this paper, we investigate a class of efficient extensions of the Myerson value for hypergraph games and provide a characterization of $E^{w} \mathrm{My}$. When restricting hypergraph games to graph games, we obtain the conclusion of van den Brink et al. and Casajus as an immediate consequence. Further research can consider degree and probabilistic hypergraph efficient extensions of the Myerson value.

\section{Data Availability}

The data used to support the findings of the study are available from the corresponding author upon request.

\section{Conflicts of Interest}

The authors declare that they have no conflicts of interest.

\section{References}

[1] R. Branzei, D. Dimitrov, S. Tijs et al., Models in Cooperative Game Theory, Springer, Berlin, Germany, 2008.

[2] R. B. Myerson, "Graphs and cooperation in games," Mathematics of Operations Research, vol. 2, pp. 225-229, 1977.

[3] L. S. Shapley, "A value for n-person games," Contributions to the Theory of Games (AM-28), Princeton University Press, Princeton, NJ, USA, pp. 307-318, 1953.

[4] R. B. Myerson, "Conference structures and fair allocation rules," International Journal of Game Theory, vol. 9, pp. 169-182, 1980.

[5] M. Slikker and A. van den Nouweland, Social and Economic Networks in Cooperative Game Theory, Kluwer Academic Publishers, Boston, MA, USA, 2001.
[6] D. Li and E. Shan, "The Myerson value on local structures of coalitions," Journal of the Operations Research Society of China, vol. 7, Article ID 461C473, 2019.

[7] D. Li Li and E. Shan, "The Myerson value for directed graph games," Operations Research Letters, vol. 48, pp. 42-46, 2020.

[8] E. Shan, J. Han, and J. Shi, "The efficient proportional Myerson values," Operations Research Letters, vol. 47, pp. 574-578, 2019.

[9] A. van den Nouweland, P. Borm, and S. Tijs, "Allocation rules for hypergraph communication situations," International Journal of Game Theory, vol. 20, pp. 255-268, 1992.

[10] E. Shan and G. Zhang, "The position value and the Myerson value for hypergraph communication situations," Frontiers of Dynamic Games Theory, vol. 19, pp. 237-250, 2018.

[11] R. van den Brink, A. B. Khmelnitskaya, and G. van der Laan, "An efficient and fair solution for communication graph games," Economics Letters, vol. 117, pp. 786-789, 2012.

[12] S. Béal, A. Casajus, and F. Huettner, "Efficient extensions of communication values," Annals of Operations Research, vol. 264 , no. 1-2, pp. 41-56, 2018.

[13] A. Casajus, "An efficient value for TU games with a cooperation structure," Universitat Leipzig, Leipzig, Germany, Working paper, 2007.

[14] X. Yi, R. Guo, and Yi Qi, "Stabilization of chaotic systems with both uncertainty and disturbance by the UDE-based control method," IEEE Access, vol. 8, no. 1, pp. 62471-62477, 2020.

[15] L. Liu, B. Li, and R. Guo, "Consensus control for networked manipulators with switched parameters and topologies," IEEE Access, vol. 9, pp. 9209-9217, 2021.

[16] T. Hou, Y. Liu, and F. Deng, "Stability for discrete-time uncertain systems with infinite Markov jump and time-delay," Science China-Information Sciences, vol. 64, pp. 1-11, 2021. 